Julia Riedel • David Buttelmann · Josep Call • Michael Tomasello

\title{
Domestic dogs (Canis familiaris) use a physical marker to locate hidden food
}

Received: 19 October 2004 / Revised: 22 March 2005 / Accepted: 10 April 2005 / Published online: 22 April 2005

(C) Springer-Verlag 2005

\begin{abstract}
Dogs can use the placement of an arbitrary marker to locate hidden food in an object-choice situation. We tested domestic dogs (Canis familiaris) in three studies aimed at pinning down the relative contributions of the human's hand and the marker itself. We baited one of two cups (outside of the dogs' view) and gave the dog a communicative cue to find the food. Study 1 systematically varied dogs' perceptual access to the marker placing event, so that dogs saw either the whole human, the hand only, the marker only, or nothing. Follow-up trials investigated the effect of removing the marker before the dog's choice. Dogs used the marker as a communicative cue even when it had been removed prior to the dog's choice and attached more importance to this cue than to the hand that placed it although the presence of the hand boosted performance when it appeared together with the marker. Study 2 directly contrasted the importance of the hand and the marker and revealed that the effect of the marker diminished if it had been associated with both cups. In contrast touching both cups with the hand had no effect on performance. Study 3 investigated whether the means of marker placement (intentional or accidental) had an effect on dogs' choices. Results showed that dogs did not differentiate intentional and accidental placing of the marker. These results suggest that dogs use the marker as a genuine communicative cue quite independently from the experimenter's actions.
\end{abstract}

Keywords Dogs · Social cognition · Object choice task . Human communicative cues

\section{Introduction}

Domestic dogs have evolved specialized cognitive skills for reading human social-communicative signals. This is

J. Riedel $(\triangle)$ · D. Buttelmann · J. Call · M. Tomasello

Max Planck Institute for Evolutionary Anthropology,

Deutscher Platz 6, D-04103 Leipzig, Germany

e-mail: riedel@eva.mpg.de

Tel: +49-0341-3550211

Fax: +49-0341-3550444 / +49-0341-3550299 most readily apparent in the so-called object choice experimental paradigm in which a human hides food in one of several distinct locations and then gives a signal to indicate for the dog which one contains food-with several different control procedures used to ensure that subjects cannot locate the food without such a cue (e.g., by smell). In many different studies dogs have shown the ability to use a number of different kinds of cues to locate the hidden food at above chance levels (Hare et al. 1998; Miklósi et al. 1998; Hare and Tomasello 1999; Agnetta et al. 2000; Soproni et al. 2001, 2002). These include such things as: (1) a human pointing to the target location (including pointing with the opposite hand with finger located at midline, equidistant from potential food locations); (2) a human gazing to the target location (dog either sees head turn or not); and (3) a human bowing or nodding to the target location. Many dogs are even skillful when a human walks toward an empty container while pointing to the target container at a different location.

One especially important condition in one of these studies involved the use of an arbitrary marker. Agnetta et al. (2000) indicated the location of the hidden food for dogs by placing a small sponge in front of the container with food. This is an especially important condition for two reasons. First, the cue is totally novel. Thus, while it is easy to imagine that dogs could have evolved specialized skills for following human pointing and eye gaze direction in various contexts - as in the majority of conditions in object choice studies - it is much less easy to imagine them evolving skills related specifically to physical markers. Second, great apes consistently have difficulties in using a physical marker to locate hidden food (Tomasello et al. 1997). Indeed, Hare et al. (2002) directly compared chimpanzees and domestic dogs in this task and found that domestic dogs were much superior. Even young dog puppies with very little human experience were successful in the task, whereas adult wolves were not-suggesting that dogs' special skills, however they might be characterized, evolved during the domestication process.

The question thus arises how dogs understand and use an arbitrary marker as a cue to food's location. The study 
of Agnetta et al. (2000) actually had 4 different conditions involving a marker. In one the dog watched the human place the marker in front of the target location, with the human looking at the location as she placed the marker (Place + Gaze). In another the dog watched the human placing the marker, but in this case the human stared straight ahead as she placed it (Place Only). In another the human raised an occluder before placing the marker so that the dog saw the following sequence: occluder raised; human holds up marker above occluder for dog to see; human lowers marker and places it on target location, with the dog not able to see the placement process (dog only sees human head and shoulders; no arm, hand, or marker during placement) (Occlude Placing). Dogs were above chance in locating the food in all of these conditions.

The fourth condition was a bit different. In this condition the dog was out of the room while the human placed the marker. When the dog entered the room it simply saw two containers, one with a sponge in front of it (human sitting behind the two containers, as in all of the other conditions). Dogs were administered this Marker Only condition two times: once before they started with the other conditions and once after they had completed them. Dogs performed at chance levels in this task at both administrations. One interpretation would be that this poor performance actually indicates that dogs understand the communicative significance of the marker. That is, they understand the sponge as a communicative sign when they observe a human use it in the proper context, but when they simply see it sitting in front of a container-without having seen a human place it-the sponge has no communicative significance. Another interpretation would be that this poor performance indicates that dogs do not really understand the communicative significance of the marker because, since this was a within subject design and dogs also experienced the Marker Only condition again at the end of the study, they knew that the game was that the human would give some sign to help them find the food - and so seeing the human actually place the sponge should be unnecessary.

It thus seems that dogs in this task need to know that a human placed the marker in order to see it as a communicatively significant sign. But note that in the Occlude Placing condition they did not actually observe the placing process either; they simply observed the human hold up the sponge for them and then perhaps move her shoulders in some subtle ways - and then they saw the marker in front of one of two buckets. Their good performance in this condition apparently means that this was sufficient information to indicate that the human had placed the marker (for them). An interesting set of questions thus arise around the issue of what human behavior dogs need to experience in association with the marker before they see the placement process as a communicative sign indicating the food's location. For example, it would be interesting to know how dogs would do in this task if they simply saw a hand place the marker without seeing any other parts of the human's body. Further, would they see the marker as a communicatively significant sign if the human placed it on a bucket but then removed it before they had to choose (a condition that would also test whether they are simply attracted to the marker)? Also interesting is the question of what dogs would infer if the human actually touched both locations but left the marker on only one-so that the placement of the marker is observed but it in a sense competes with a simple touching cue (which has been shown in other studies to be effective; Hare et al. 2002). Finally, it would also be interesting to know if the way in which the human places the marker is important, with special reference to the issue of whether the human places it intentionally or by accident (see Call and Tomasello 1998, for a similar study with apes and human children).

The current study, therefore, comprises three experiments with dogs using the object choice paradigm with a marker. In the first we systematically varied dogs' perceptual access to the event of the human placing the marker, such that sometimes the dog saw only the hand doing the placing or just the face/shoulders of the human and so forth. We also sometimes removed the marker before the dog chose, to see if the marking process retained its communicative import even without the physical marker. In the second study the human touched both hiding locations, in turn, leaving the marker either on the first one touched or the second one touched, enabling us to determine if the marker provided the dogs with information above and beyond the human's behavior. And in the third study we placed the marker on both containers before the dog chose (removing it before the choice) - with one of the placements occurring accidentally ("Whoops!") and the other occurring intentionally ("Ja!").

\section{Study 1: Different parts of the marking process visible}

\section{Methods}

\section{Subjects}

Sixty-four domestic dogs (Canis familiaris) of various breeds and ages participated in the study. There were 36 females and 28 males that ranged in age from 9 months to 11 years (mean $=4.35 \mathrm{yrs}, \mathrm{SD}=2.46 \mathrm{yrs}$ ). Just under half the dogs were mongrels, and most of the others were some breed of working dog (herding, hunting, and others). All dogs were recruited by phone from owners in a mediumsized German city. Some dogs had participated in previous experiments, but none using markers.

During the study we recruited more than 64 dogs to maintain a good sample size and some dogs would not perform the test. Fewer than four dogs did not pass the warm-up, so they never started with the experimental conditions. For each condition we had fewer than 5 dogs which passed the warm-up but then after some trials simply did not perform anymore, due to the lack of attention and less food motivation.

\section{Procedure}

Testing was done during September and October 2002 in a quiet room of approximately $10 \mathrm{~m}^{2}$, without owners being 
present. The basic task had the same structure in all four experimental conditions. On each trial, one experimenter (E1) stood behind the dog and held it by the collar, with its two forelegs on a marked spot $1.5 \mathrm{~m}$ in front of the test table. A second experimenter (E2) sat behind this table on a small stool $25 \mathrm{~cm}$ high and prepared for the coming trial by showing a piece of food (dog treat) to the subject, raising a horizontal barrier $(120 \times 45 \mathrm{~cm})$ in front of the table, and then behind this barrier hiding the treat under one of two identical plastic cups (red coloured, $d=8.5 \mathrm{~cm}$, $h=8 \mathrm{~cm}$ ). The cups were placed on the table $90 \mathrm{~cm}$ apart and turned upside down so that the dogs could not see the food. To equalize the olfactory cues potentially provided by the food inside the cups, both cups had been in contact with food pieces before the experiment began.

Each dog participated first in five warm-up trials in which it observed E2 hiding the food, and then was released to make its choice. Virtually all dogs met the criterion of 4 of 5 correct immediately; the few who did not were given additional warm-up trials until they got four out of five consecutive trials correct. After this warm-up phase, testing proper began. E2 showed a piece of food to the subject and called its name to get the subject's attention, then E2 hid the reward under one of the cups. The piece of food was placed in the middle of the table and both cups were moved towards the food, but only one cup captured the food and dragged to its final destination. Than E2 administered one of the four following conditions (see Fig. 1):

All. E2 lowered the horizontal barrier and obtained the dog's attention by showing it a marker (black and white colored piece of wood, $11 \times 7 \times 3 \mathrm{~cm})$. He then placed the marker on top of the cup with food, looking to the cup as he placed the marker.

Hand only. E2 lowered the horizontal barrier, but then put up another one that hid his entire body and the middle part of the table (vertical barrier, $90 \times 53 \mathrm{~cm}$ ). The dog could thus see only the two ends of the table with the cups on top of them $(26 \mathrm{~cm}$ on each side of the table). Then, without showing the marker to the dog and without obtaining the dog's attention, E2 placed the marker on top of the cup with food. All that the dog saw was a hand emerging from behind the barrier and placing the marker, but not E2's gaze direction.

Marker only. E2 did not immediately lower the horizontal barrier used to occlude the hiding process. With the horizontal barrier still up, E2 obtained the dog's attention by showing it the marker, and then placed the marker on top of the cup with food, looking to the cup as he placed the marker. The dog was unable to see either the cups or the actual marker placement. It could only see the gaze cue of the experimenter and the movement of his shoulder.

Nothing. E2 did not immediately lower the horizontal barrier used to occlude the hiding process, and without obtaining the dog's attention he also raised the vertical barrier that occluded him as well. Thus, the dog was unable to see any element of the placement process except the marker on top of the cup after E2 lowered the two barriers.

After E2 had given his cue, he resumed a waiting position, looking straight ahead and E1 released the dog to make its choice.
Fig. 1 Graphic depiction showing the experimental set-up of the four conditions in study 1

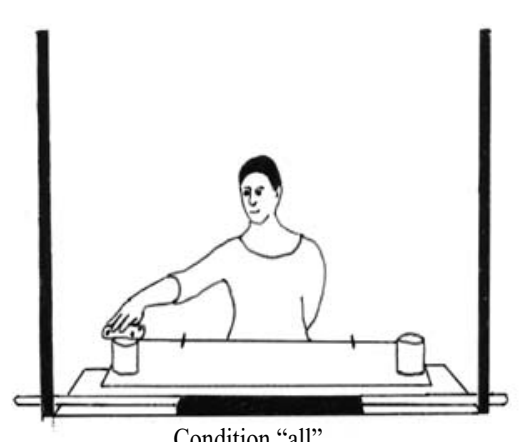

Condition "all"

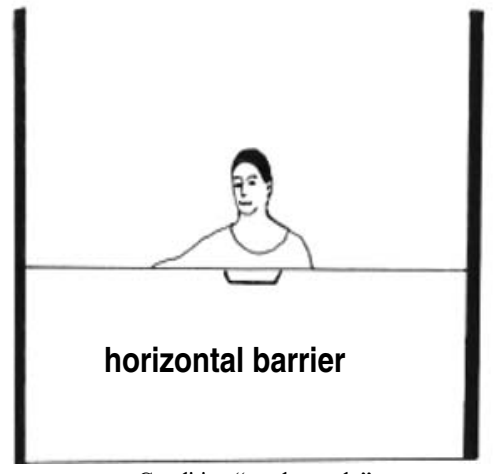

Condition "marker only"

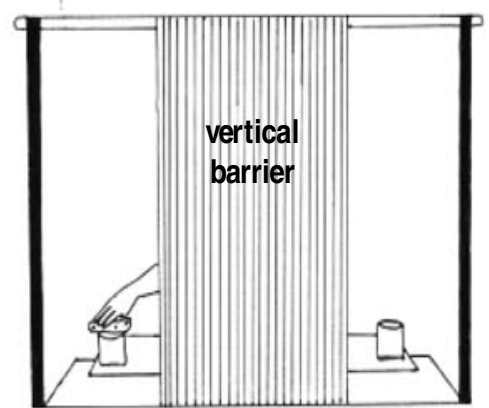

Condition "hand only"

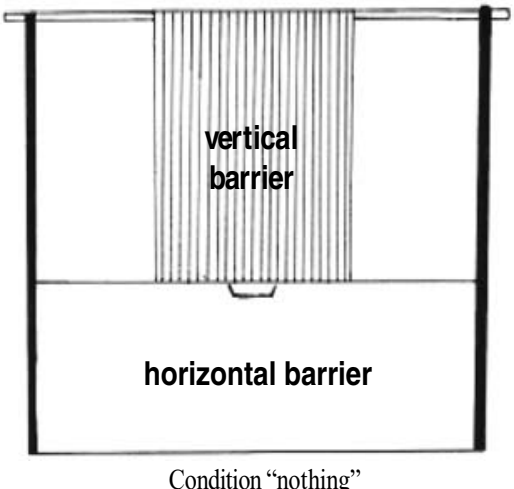


The experimental design was between-subjects, with 16 subjects randomly assigned to each of the four conditions. Each subject received 18 trials in its assigned condition, all in a single session (with a short break after 12 trials). The side on which the food was hidden was semirandomized, with the stipulation that it never occurred more than twice in a row on the same side. After these 18 trials, an additional experimental manipulation was introduced. Each subject received six additional trials in each of the four conditions mentioned above except that E2 removed the marker from the cup after $3 \mathrm{~s}$ had elapsed since its placement, these were called 'marker gone' (at the time of the dogs choice) trials. This means that dogs always saw the hand removing the marker from the baited cup and E2 looking at the cup while the removal took place. The dog was released only after this removal, and so its choice was made with no cue immediately available.

As in previous studies, dogs almost always made their choices immediately by approaching one of the cups and nudging it with the nose. Since choices were totally unambiguous, scoring was done simply by the two experimenters agreeing on the choice and writing it down on the spot (in past studies of this type inter-observer reliabilities have always approached $100 \%$ ). If the dog chose the correct cup, it was allowed to eat the food; if it chose the incorrect cup, E2 took the food from the correct cup with the dog watching and put it back in a bag.

We used ANOVA and $t$-test to make comparisons across conditions and one-sample t-tests (with $50 \%$ expected probability) to assess the deviation from chance responding. All tests were two-tailed.

\section{Results}

Figure 2 shows the mean percent correct responses in both the 18 'marker present' experimental trials and the six 'marker gone' (at the time of the dogs choice) experi- mental trials. For the 'marker present' experimental trials, each condition was first compared to chance. All conditions except the 'Marker Only' condition $(t(15)=2.09$, $p=0.055$ ) were above chance (All: $t(15)=9.32, p<0.001$; Hand Only: $t(15)=8.20, p<0.001$; Nothing: $t(15)=2.38$, $p=0.031)$. The analysis of the effect size for the "marker only' condition shows a Cohen's medium effect $(d=0.52)$, so with a sample size of 16 subjects we can not detect significance.

These four conditions may be characterized by the factorial combination of two factors: whether or not the dog can see the hand and whether or not the dog can see the human's body and gaze as he places the marker [All $=++$; Hand Only $=+-$; Marker Only $=-+$; Nothing $=-$ ]. To compare these factors and their possible interaction, an overall 2 (hand: visible/not visible) $\times 2$ (body: visible/not visible) ANOVA was conducted. The only effect found was for visibility of the hand, $F(1,15)=80.91, p<0.001$. Dogs performed significantly better when they could see the hand placing the marker.

In the 'marker gone' experimental trials, all conditions were higher than would be expected by chance (All: $t$ $(15)=6.58, p<0.001$; Hand Only: $t(15)=3.62, p=0.003$; Marker Only: $t(15)=2.78, p=0.014$; Nothing $t(15)=3.58$, $p=0.003$ ). An overall 2 (hand: visible/not visible) $\times 2$ (body: visible/not visible) ANOVA revealed no significant main effects or interactions. To compare the two types of experimental trials ('marker present' and 'marker gone' at the time of the subjects choice) we conducted a repeated measurement ANOVA with the following factors: 2 (hand: visible/not visible) $\times 2$ (body: visible/not visible) $\times 2$ (marker: 'marker present' trials/'marker gone' trials). There was a significant main effect for the visibility of the hand, $F(1,15)=24.87, p<0.001$, and a significant hand $\mathrm{x}$ marker interaction, $F(1,15)=5.75, p=0.03$, such that the visibility of the hand was more important in the "marker present' than in the 'marker gone' trials. There were no other reliable main effects or interactions.
Fig. 2 Mean correct responses in percent shown by each of the four testing groups in the 'marker present' and 'marker gone' at the time of the dogs choice conditions with SE for each group

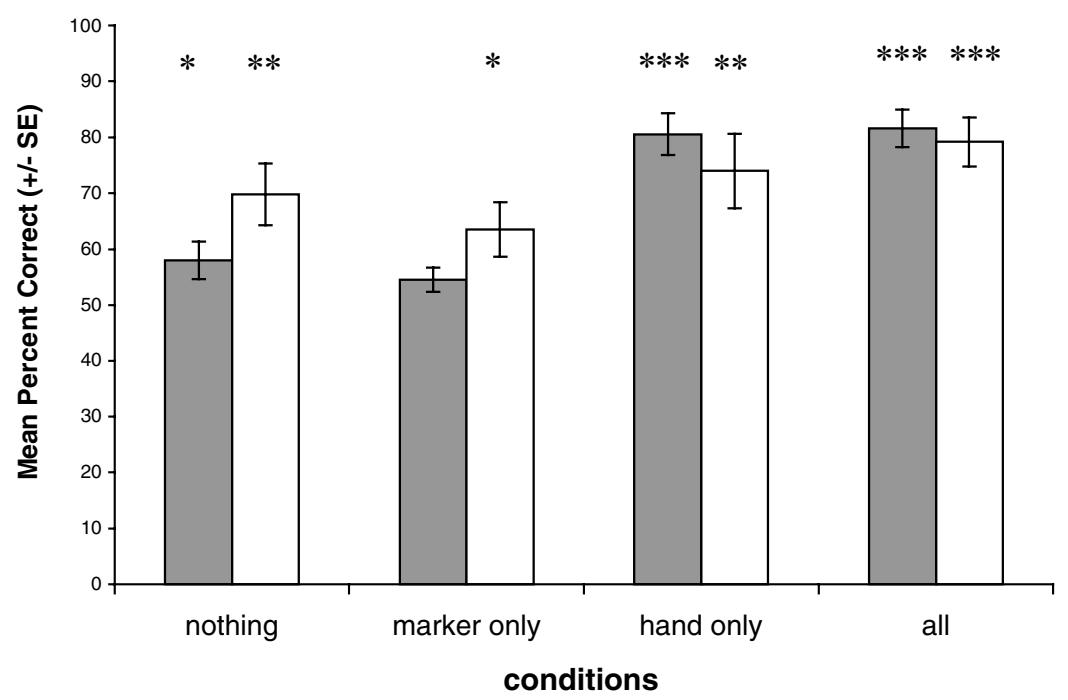

$\square$ marker present $\square$ marker gone 
To test for learning during the experiment, the first six trials of the 'marker present' experimental conditions were compared to the last six trials using a paired t-test for each condition. No significant effects were found (no test statistic above 1).

We conducted first trial analyses for the four "marker present' conditions together, 43 dogs were correct in their first choice, 21 subjects were wrong. A binomial test against chance (Probability $=0.5$ ) showed a significant effect $N=64 p=0.008$.

There were also no reliable differences due to breed or sex.

\section{Discussion}

In this study, dogs used basically all of the marker-based cues to find the hidden food. When the marker stayed on the cup, they used 3 of the 4 cues. The non significant 'Marker Only' cue was when the dog saw the experimenter hold up the food to begin the trial, did not witness the hiding process (though it did see E2 hold up the marker and look in a direction behind the occluder), but only saw the marker on the cup when it came time to choose. But a very similar cue was effective in the study of Agnetta et al. (2000), and the same exact cue was effective in the current study when the experimenter removed the marker before the moment of choice in the 'marker gone' trials. In general, then, the basic finding is that dogs were skillful with all of the marker-based cues.

This was even true of the condition 'Nothing' in which the dog did not witness any part of the hiding process (e.g., did not see E2 hold up the marker or look in any direction), but had information only at the very end as it saw the marker on top of the cup. In a similar condition, Agnetta et al. (2000) found chance performance in dogs. The main difference is that in that study the dog was out of the room both during the baiting process and when the experimenter placed the marker-and so did not see E2 with the food at all. One interpretation of the discrepancy between these findings is that in the current study, dogs always saw E2 in his place behind the table, with food, at the beginning of the trial, and so when they saw the marker on the cup inferred that he had placed it - whereas in the Agnetta et al. (2000) study this inference was more difficult because dogs were not even in the room when the baiting and marking took place.

One could argue that successful performance may be based on using the experimenter's gaze. However, this was not the case because there was also a high performance in the 'Hand Only' and 'Nothing' conditions in which dogs were prevented from seeing the experimenter's gaze direction. Alternatively, one could argue that dogs based their decision on the auditory cue produced by the placement of the marker. However, recall that the marker was placed carefully and had a padded surface to prevent the production of auditory cues. More importantly, the presence of auditory cues would not explain the observed differences across conditions because the marker placement was identical in all conditions.

Comparison among the cues revealed that the very best conditions were ones in which the dog could see the hand placing the marker on the cup, and this was especially so when the marker remained on the cup at the time of choice (interaction between hand and marker in the overall ANOVA). One possible reason for this interaction is that in the conditions in which the marker was gone at the moment of choice, the dog always witnessed the removal process and so in this case the hand went to the correct cup (to remove the marker) just before the dog chose. So it is possible that the hand is important in these conditions as well.

In general, it is important that removing the marker before the dog chose overall had no detrimental effect on performance. This is a crucial point because it demonstrates very clearly that dog's behavior in these tasks did not derive from their attraction to the marker itself. Nevertheless, given that the dog saw the removal process, it is true that of the eight experimental conditions in the study as a whole, the dog saw the hand with a marker in it on top of the correct cup (either placing or removing) in six of these. Interestingly, the two conditions in which dogs did not see this configuration ('Nothing' and 'Marker Only' in main trials) were the two lowest conditions numerically.

It thus seems plausible that attention to both the marker and hand contributed to dogs' success in this experiment. In a second experiment, therefore, we attempted to assess dogs' understanding of the marker independent of the hand by having the experimenter always touch both cups during the marking process, but then leave the marker on top of only one of them.

\section{Study 2: The role of the marker}

\section{Methods}

\section{Subjects}

Sixteen domestic dogs (Canis familiaris) of various breeds and ages participated in this study. There were 7 males and 9 females that ranged in age from one to nine years (mean $=3.5 \mathrm{yrs}, \mathrm{SD}=2.25 \mathrm{yrs}$ ). About one-third of the dogs were mongrels, and most of the others were some breed of working dog (herding, hunting, and others). Again dogs were recruited by phone from owners in a medium-sized German city. None of the dogs had participated in the first study.

\section{Procedure}

Testing was done between January and April 2003 in the same room as the first study, without owners present. The warm-up trials were conducted exactly as in the first study. The basic procedure of the experimental trials and the materials were the same as in the first study also: E2 showed 
the dog the food to get its attention, hid the food in one of two cups behind a barrier, lowered the barrier, gave a cue, and then gave the dog a chance to choose a cup. What differed, of course, were the two experimental conditions:

Marker vs. hand. After baiting E2 lowered the barrier obtained the dog's attention by showing it the marker. He then placed the marker on top of the cup with food, looking to the cup as he placed the marker. Leaving the marker in its place, he then briefly touched the other cup on its top and looked at it. After placement, he resumed a waiting position, looking straight ahead.

Marker touches both. After baiting E2 lowered the barrier, obtained the dog's attention by showing it the marker, and then briefly touched the top of the cup without food using the marker, and then the cup with food, looking at both cups as he placed the marker. Leaving the marker there, he resumed a waiting position, looking straight ahead.

The experimental design was within-subjects, with each subject receiving 4 trials in each condition, all in a single session. The order of conditions was randomized and the side on which the food was hidden was semi-randomized, with the stipulation that no condition or side ever occurred more than twice in a row. Scoring was done by the two experimenters agreeing on the choice and writing it down on the spot. If the dog chose the correct cup, it was allowed to eat the food; if it chose the incorrect cup, E2 took the food with the dog watching and put it back in a bag. We analyzed the data as in the previous study.

\section{Results}

Table 1 shows the number correct (out of 4 ) in each condition for each subject.

Table 1 Number of trials correct (out of 4) for each subject in each condition

\begin{tabular}{llll}
\hline Subject & Breed & Both marked & Marker vs. Hand \\
\hline Lisa & Border Collie & 3 & 4 \\
Sunny & G. Retriever & 2 & 3 \\
Ronja & G. Retriever & 2 & 2 \\
Charly & G. Retriever & 2 & 2 \\
Toby & G. Retriever & 2 & 3 \\
Ricky & G. Retriever & 2 & 3 \\
Bajinia & Ridgeback & 3 & 2 \\
Ambula & Ridgeback & 1 & 4 \\
Willy & Boxer & 2 & 3 \\
Balou & Schapendoes & 2 & 4 \\
Sally & Terrier & 1 & 2 \\
Akira & Mongrel & 2 & 3 \\
Lotte & Mongrel & 2 & 2 \\
Tina & Mongrel & 2 & 2 \\
Tina2 & Mongrel & 2 & 2 \\
Winnie & Mongrel & 1 & 4 \\
Total & & $1,9448,44 \%$ & $2,8270,31 \%$ \\
\hline
\end{tabular}

The 'Marker vs. Hand' condition significantly differed from chance, $t(15)=3.90, p=0.001$ whereas the 'Marker Touches Both' condition did not, $t(15)=0.44$, $p=0.669$. Moreover, dogs performed significantly better in the 'Marker vs. Hand' condition compared to the 'Marker Touches Both' condition, $t(15)=3.22, p=0.006$. No significant effects were found for breed or sex.

\section{Discussion}

Dogs again seemed most strongly influenced by seeing the hand with the marker in it on top of the cup. Thus, in the 'Marker vs. Hand' condition, they saw the marker in the hand only on top of the correct cup, and so they were quite good (correct over $70 \%$ of the time), ignoring the empty hand touching the other cup. In contrast, in the 'Marker Touches Both' condition in which they saw the marker in the hand on top of both cups in turn, they performed at chance (48\%) - even though the marker was sitting on the correct cup at the moment of choice. One could argue that successful performance in the 'Marker vs. Hand' condition was caused by a primacy effect, that is, dogs targeted the cup where the marker first rested on. However, this would not explain the chance performance in the 'Marker Touches Both' condition. Indeed, a primacy effect would have produced a below chance performance, not the observed chance performance, because the cup marked first was always incorrect in this condition. It thus seems, once again, that neither the marker by itself, the hand by itself or some primacy effect is sufficient to explain these findings, but rather dogs are looking to see the human place the marker. In the next study we investigated how sensitive dogs were to the way the marker was deposited on the cup.

\section{Study 3: Purposeful vs accidental placing}

The first two studies concerned where the marker was placed and what the dog could see of the placing process. The third study, modeled on that of Call and Tomasello (1998) with apes, concerned the manner in which the marker was placed — either deliberately or by accident.

\section{Methods}

Subjects

The same dogs that participated in Study 2 also participated in Study 3, after an approximately 15 min break.

\section{Procedure}

The basic procedure was the same as in the first two studies: E2 showed the dog the food to get its attention, hid the food in one of two cups behind a barrier, lowered the barrier, gave a cue, and then gave the dog a chance to choose 
a cup. The apparatus and, of course, the experimental conditions were different. On the table, behind each cup (from the subject's point of view), was a small "tower", approximately $5 \mathrm{~cm}$ taller than the top of the cup. After baiting the cups E2 lowered the barrier, obtained the dog's attention by showing it the marker. The marker for the study was a small beanbag toy. The beanbag was placed on the tower behind a cup, and E2 then either pushed it onto the cup with one finger purposefully—saying: "Ja!" (german word for yes) while directing his body and gaze towards the marker and the cup, or turned his head away from the marker and towards the ground (with sides randomized) and "accidentally" raised/bumped the table with his knees so that the beanbag fell off the tower onto the cup-saying "Whoops!" as an additional cue to the purpose of the action. There were two conditions depending on the order of the type of marking:

Intention First. E2 placed the marker deliberately on top of the cup with food, paused, removed it, and then knocked it "accidentally" onto the other cup, and removed it (into his lap beneath the table).

Accident First. E2 knocked the marker "accidentally" on top of the cup with food, paused, removed it, and then placed it deliberately on the other cup, and removed it (into his lap beneath the table).

After this, E2 resumed a waiting position, looking straight ahead, and E1 released the dog to make a choice. Thus, for each trial the marker was placed, in turn, on both cups, once accidentally and once purposefully, and always removed before the dog made its choice.

The experimental design was within-subjects, with each subject receiving 4 trials in each condition, all in a single session. The order of conditions was counterbalanced across subjects, and the side on which the food was hidden was semi-randomized such that no side ever occurred more than twice in a row. The scoring procedure and statistical analyses were the same as those in the previous studies.

\section{Results}

The dogs in this study did not choose the cup marked intentionally at greater than chance levels (mean $=4.4$ out of 8$), t(15)=1.46, p=0.16$. The dogs were not choosing randomly, however. Rather, they selected the cup marked last significantly more often than the cup marked first, (mean last $=4.75, \mathrm{SE}=0.27$; mean first $=3.25, \mathrm{SE}=0.27$ ) $t(15)=2.82, p=0.013$. Finally, we analysed the results of the dogs' first choice, so the first accident that happens for the subject, 8 dogs were correct and 8 dogs were not, there is no tendency that the dogs perform better in the first accident that happens.

\section{Discussion}

In this study dogs saw the marker being placed on both cups before they made their choice. The question was whether they would pay attention to the manner in which that placing was conducted, either intentionally or accidentally. They did not, but rather they simply went for the last cup marked. It would thus seem that, using the current methodology at least, dogs do not distinguish between intentional and accidental actions.

It is not clear why they chose to go for the last cup marked. One possible explanation is that the trial took some time to execute, what with placement, removal, placement, removal. And so, given that the dogs were not good at distinguishing the different types of placing, they just went for the most salient cue, the last one seen.

\section{General discussion}

The current studies succeeded in outlining some of the parameters of dogs' performance in using the marker as a communicative cue. In particular, they allowed us to pin down the relative contributions of the human's hand and the marker in a communicative situation. The first study showed that dogs performed better when seeing the hand place the marker than seeing the marker alone. Nevertheless, dogs were able to use the marker both without seeing the actual placing or with the marker absent at the time of choice.

The second study served to reinforce the importance of the marker independent from the hand. Dogs selected the correct cup with the marker on top even if both cups had been touched by the hand before the choice, but they did not do so if the marker (and the hand) had visited both cups. The third study confirmed this result by showing that subjects were unable to choose the correct cup when the marker had been on both cups and the hand had only been involved in the placement on one of them.

Looking at the data across studies revealed that dogs succeeded in those conditions in which only one of the two cups had been associated with the marker (i.e., study 1: all conditions, study 2: 'Marker vs. Hand') but failed those conditions in which the marker had been associated with both cups (study 2: 'Marker Touches Both', study 3: both conditions). In contrast, the involvement of the hand did not have such an effect on performance. Dogs succeeded in some conditions when the hand was directed to a single cup only (study 1) but failed in others (study 3 ). Similarly, dogs passed some conditions in which the hand was directed to both cups (study 2: 'Hand vs. Marker') but failed others ('Marker Touches Both'). This means that dogs were attaching more importance to the marker than to the hand that placed the marker, although the presence of the hand could boost performance when it appeared together with the marker in one cup (study 1).

The most fundamental question arising from dogs' generally excellent performance in using a marker to find hidden food is whether they actually understand this cue communicatively, or whether, alternatively, they are succeeding in some other way. Control conditions in previous studies have demonstrated conclusively that they are not using smell (e.g., Hare et al. 1998; Agnetta et al. 2000). The "marker 
gone' trials in the first of the current studies demonstrated further that they are not simply attracted to the current physical presence of the marker and that the dogs do not need the presence of the marker to choose correctly.

Moreover, when the marker was present they directed their actions to the cup, not to the marker. In fact, we have not observed any difference in the way dogs behave when those have been single out by the marker or by a pointing gesture.

Neither in this study nor in other studies do dogs need time to learn to use the marker as a discriminative cue, as they are skillful from the very first trials. So, on the face of it, dogs seem to understand the marker as a communicative cue.

Previous studies have shown that dogs are very good at using such direct human communicative behaviors as pointing and looking as cues that indicate for them the location of hidden food, and they are good at this even as young puppies when they have had varied little opportunity to learn such things from their interactions with humans (Hare et al. 2002). It is thus possible that dogs have evolved an adaptive specialization for reading human directional behaviors, such as looking and pointing, and these accounts for their good performance. But the physical marker, as used in the current study, is a novel communicative cue for which no plausible evolutionary story can be told. This means that dogs' adaptation, whatever it may be precisely, supports very general skills of reading human communicative behavior-even when they must do this indirectly by inferring that the human has placed a marker on an important location.

Comparing our current results on the use of the marker to those of previous studies with children and apes suggests that dogs and 30- to 36-month-old human children can use novel cues that they have had no previous opportunity to learn in this same way, whereas the same is not true for chimpanzees and orangutans (Tomasello et al. 1997). Note that all great apes that had been trained on the marker in a previous task performed at above chance levels whereas the untrained apes performed at chance level. This poor performance occurred even though they were tested under conditions similar to the "all" condition in the current study, which was the easiest condition for dogs. Future studies could investigate how children and trained apes react to the presentation of partial information regarding the placement of the marker as we did in this study for dogs. Another avenue of future inquiry is to investigate the ways in which dogs do and do not use other kinds of novel human-given communicative cues. Especially important would be testing the skills of human-naive puppies' in the use of gestures such as pointing and arbitrary cues such as the marker to see how the different types of cues appear during ontogeny.

This study confirmed previous findings showing that dogs are capable of using an arbitrary marker as a communicative cue (Agnetta et al. 2000; Hare et al. 2002). The two conditions that are problematic to some degree are: (1) the 'Nothing' condition in the 'marker present' trials, which was just above chance in the current study, but in a slightly altered form was at chance in the Agnetta et al. study; (2) the 'Marker Only' condition in the 'marker present' trials which was at chance level in the current study (although in a slightly altered form above chance in the Agnetta et al. study). It is likely that in this first of these conditions the way the cue was given in the current study was more informative than in the Agnetta et al. study in which the dog was out of the room for the entire baiting and marking process. In the second of these, it is not clear if perhaps there was some subtle difference between the current study and the Agnetta et al. study (e.g., a slightly higher or lower barrier) that might have provided less information here. In any case, the current hypothesis is that to the degree that a dog was able to infer from observed information (they saw the human with the food, the raising of the barriers and the marker on the cup) that the human had placed the marker, to that degree was it successful.

Information about the removal process also helped boost dogs' performance to some degree in Study 1 (and it may have also played a role in our inability to find significant differences between a marker placed on the correct cup deliberately and one placed there accidentally). However, negative results are never definitive, and so since this is the first study of its kind-we know of no other studies specifically testing whether dogs discriminate intentional actions from other kinds of actions-we can only say that in the current study dogs did not seem to make this discrimination. Interestingly, great apes in the study of Call and Tomasello (1998) did make this discrimination, and in the study of Call (2004) they used various physical/causal cues (e.g., noises) to infer where hidden food might be. But in other studies, great apes do not use a human-placed marker as a communicative cue indicating the location of hidden food (see Call and Tomasello 2003, for a review, and Hare et al. 2002, for a direct comparison between the species). One possibility is that great apes have mainly evolved skills for making inferences about their causal and social worlds, whereas dogs have been specifically selected during the domestication process to be sensitive to human-produced communicative cues (see Bräuer et al. submitted, for some suggestive evidence in this direction).

In conclusion, dogs used an arbitrary marker as a communicative cue. They attached more importance to this cue than to the hand that placed the marker, although the presence of the hand boosted performance when it appeared together with the marker and the marker was not associated with the other cup.

Acknowledgements The authors wish to thank Paul Harris for the basics of the idea for study 2 and we also thank the dog owners for their co-operation. The reported experiments comply with all laws of the country (Germany) in which they were performed

\section{References}

Agnetta B, Hare B, Tomasello M (2000) Cues to food location that domestic dogs of different ages do and do not use. Anim Cogn 3:107-112

Call J (2004) Inferences about the location of food in the great apes. J Comp Psychol 118:232-241 
Call J, Tomasello M (1998) Distinguishing intentional from accidental actions in orangutans (Pongo pygmaeus), Chimpanzees (Pan troglodytes), and human children (Homo sapiens). J Comp Psychol 112:192-206

Call J, Tomasello M (2003) Social Cognition. In: Maestripieri D (ed) Primate psychology: the mind and behavior of human and nonhuman primates. Harvard University Press, Cambridge, MA, pp 234-253

Hare B, Call J, Tomasello M (1998) Communication of food location between human and dog. Evol Commun 2:137-159

Hare B, Tomasello M (1999) Domestic dogs (Canis familiaris) use human and conspecific social cues to locate hidden food. J Comp Psychol 113:173-177
Hare B, Brown M, Williamson C, Tomasello M (2002) The domestication of social cognition in dogs. Science 298:1634-1636

Miklósi Á, Polgárdi R, Topál J, Csányi V (1998) Use of experimenter-given cues in dogs. Anim Cogn 1:113-121

Soproni K, Miklósi Á, Topál J, Csányi V (2001) Comprehension of human communicative signs in pet dogs (Canis familiaris). $\mathrm{J}$ Comp Psychol 115:122-126

Soproni K, Miklósi Á, Topál J, Csányi V (2002) Dogs (Canis familiaris) responsiveness to human pointing gestures. J Comp Psychol 116:27-34

Tomasello M, Call J, Gluckman A (1997) Comprehension of novel communicative signs by apes and human children. Child Dev 6:1067-1080 